\title{
GMRT observations of the protostellar jet associated with IRAS 16547-4247
}

\author{
Josep M. Masqué ${ }^{1}$, Solai Jeyakumar ${ }^{1}$, Miguel A. Trinidad ${ }^{1}$, Tatiana Rodríguez-Esnard ${ }^{1}$, \& \\ CH Ishwara-Chandra ${ }^{2}$
}

\begin{abstract}
We report continuum GMRT observations aimed to explore the behavior of the jet associated with IRAS 16547-4247 at very low frequencies (325 MHz and $610 \mathrm{MHz}$ ). The obtained maps reveal an elongated morphology in the SE-NW direction. In addition, the $610 \mathrm{MHz}$ map shows three knots associated to the elongated morphology that seems to correspond to the triple radio source identified as the jet seen at higher frequencies. However, at $325 \mathrm{MHz}$, although an elongated morphology is also observed, only two knots appear in the map. By comparing our knot positions at both frequencies with a precessing jet model used in a previous work, we find that the knots fall closely to the wiggling path and, hence, they likely represent shocks of the material of the precessing jet with the medium. Only the nature of the southernmost knot detected at $325 \mathrm{MHz}$ remains unclear. Besides, we found that the whole emission of the lobes is nonthermal down to very low frequencies and that the possible associated emission mechanisms work differently in in both lobes, causing the discrepancies between observed frequencies. To explain these discrepancies we investigate mechanisms such as synchrotron radiative losses in a magnetic field of $\sim 0.5 \mathrm{mG}$ in the shocks and find this possibility unlikely to occur. Alternatively, we invoke interaction with an inhomogeneous medium as the most probable scenario.
\end{abstract}

Subject headings: radiation mechanisms: non-thermal, stars: formation, ISM: jets and outflows

\section{Introduction}

Collimated winds constitute an ubiquitous phenomena in the star forming process. It is believed that they are launched at high velocities via magnetohydrodynamic proceses

\footnotetext{
${ }^{1}$ Departamento de Astronomía, Universidad de Guanajuato, Apdo. Postal 144, 36000 Guanajuato, Méx-

${ }^{2}$ National Centre for Radio Astrophysics, TIFR, Post Bag No. 3, Ganeshkhind Post, 411007 Pune, India
} ico 
occurring in an accretion disk surrounding a young protostar (Shu et al. 1987; Ouyed \& Pudritz 1997; Fendt 2006; Zanni et al. 2007). In a wide range of scales, collimated winds give rise to several observational signposts (see Bally 2016). At scales ranging from tens to hundreds of $\mathrm{AU}$ from the protostar, the wind is partially ionized and its radio emission is dominated by free-free radiation(Reynolds 1986; Anglada 1996; Anglada et al. 2018). When angularly resolved, the winds appear as elongated structures associated with the protostar and are called 'thermal radio jets' (e.g. Rodríguez et al. 1990; Girart et al. 1996). On larger scales, the interaction between the collimated wind and the surrounding cloud is detected. Such interaction can be observed as molecular outflows that are produced by the molecular material of the cloud entrained by the collimated wind (Raga \& Cabrit 1993, Raga et al. 2003. Anglada et al. 1995), or with strong shocks between the high velocity outflowing gas and the interestelar medium. At optical wavelengths, Herbig-Haro $(\mathrm{HH})$ objects are manifestations of these powerful shocks (Reipurth \& Bally 2001). Additionally, the high excitation of $\mathrm{HH}$ objets makes them emitting objects at other frequency bands (e.g. HH 80-81: Rodríguez \& Reipurth 1989; López-Santiago et al. 2013), including atomic and molecular lines (e.g. Eislöffel et al. 2000, Anglada et al. 2007; López et al. 2009). Large scale maps of star forming regions show that $\mathrm{HH}$ objects can be usually associated in chains of knots forming large $\mathrm{HH}$ systems ending up to a bow shock, which is associated with the terminal working surface of the jet (see Reipurth \& Bally 2001).

Despite the wide range of stellar masses determined for young objects, it appears that all of them have associated jets suggesting, not without controversy, that high mass protostars form under a similar mechanism than low mass protostars. However, massive protostars undergo a much intense accretion phase than low mass stars (Walmsley 1995; Pudritz et al. 2007). If the protostellar accretion is intimately bound to the launch of collimated winds, then much powerful jets should be observed in high mass star forming regions with respect to their low mass counterparts. A clear example is found in the HH 80-81 system for which projected sizes of 10.3 pc (Masqué et al.2012) and tangential velocities between 200 and 1000 $\mathrm{km} \mathrm{s}^{-1}$ have been measured (Martí et al. 1995, 1998; Masqué et al. 2015). The morphological resemblance of this jet emanating from a high mass protostar to other jets found in low mass young objects suggests that protostellar jets scale their properties depending on the mass of the powering source. This scenario is strengthened by recent observations that resolve the disk around the massive star powering the $\mathrm{HH}$ 80-81 radio jet (Girart et al. 2018).

However, the shocks observed in massive star forming regions can have different properties from those observed around low mass young objects, owing to the higher velocities in which the jet material slams on the ambient medium. Up to the date, a handful of massive YSO have been reported to present negative spectral indices at radio wavelengths (e.g. HH 80-81: Martí et al.|1993; Masqué et al.2012; L778: Girart et al.|2002, W3 (OH): Wilner et al. 
1999). This implies a significant contribution of non-thermal emission in the shocks that is usually proposed to be synchrotron emission produced by a population of ultra-relativistic electrons. In this sense, Carrasco-González et al. (2010) performed successfully a direct measure of the magnetic field strength and direction in the $\mathrm{HH}$ 80-81 jet, providing for the first time, evidence of the presence of a population of relativistic particles trapped in a magnetic field present in the jet. Recently, Ainsworth et al. (2014) reported for the first time a negative spectral index in a bow shock associated to an outflow emanating from a low-mass YSO, suggesting that relativistic particles are also present in low-power jets.

Relativistic populations of particles are easily found in the AGN jets (e.g. Blandford et al. 1982 ) and supernova remnants (e.g. Castro \& Slane 2010), where gas flows reach velocities up to a significant fraction of the speed of light. However, the material of protostellar jets moves at a speed of few hundred of $\mathrm{km} \mathrm{s}^{-1}$. For theses velocities, particle acceleration mechanisms have been proposed to work on the free electrons of the gas, such as strong shocks created by the jet in the ambient medium. In order to understand the acceleration process, detailed simulations of propagating relativistic electron beams were carried out using numerical solutions to the Fokker-Planck equation (Casillas-Pérez et al. 2016). These simulations suggest that the electron population will quickly lose most of the low energy electrons due to collisions with the ambient gas. This provides some diagnostics to be tested, especially using low frequency observations around massive young stellar objects where strong shocks with its surrounding medium are expected to be found as a consequence of protostellar activity.

IRAS 16547-4247, with a luminosity of $6.2 \times 10^{4} L_{\odot}$, is associated with a massive star forming region located at a distance of $2.9 \mathrm{kpc}$. Continuum and molecular line observations at mm wavelengths show IRAS 16547-4247 coincident with a dusty dense core of $10^{3} M_{\odot}$ (Garay et al. 2003). The same authors carried out radio observations over the region and found three components aligned in the SW direction with the outer components symmetrically separated from the central source by $10^{\prime \prime}(0.14 \mathrm{pc}$ in the plane of sky). The triple radiosource was proposed to constitute a central jet with two outer lobes representing the working surfaces of the jet with the medium. Interestingly, the spectral indices of the lobes $(\alpha=-0.61$ and -0.33 , where $\alpha$ is defined as $S_{\nu} \propto \nu^{\alpha}$ and is derived from 1 to $10 \mathrm{GHz}$, approximately) suggest the presence of non-thermal emission. Besides, Brooks et al. (2003), through IR observations, show that the collimation of the outflow persists up to $1.5 \mathrm{pc}$ from the powering source, making IRAS 16547-4247 the first case of a collimated outflow powered by an Otype star. A further study over the region using molecular lines reveals an energetic outflow associated to IRAS 16547-4247, making evident the powerful ejecta form the massive star Garay et al. 2007). However, Rodríguez et al. (2008), using two sets of $3.6 \mathrm{~cm}$ observations, do not detected proper motions along the axis of the outflow in the outer lobes, although 
they show evidence of precession.

IRAS 16547-4247 has also been proposed as a potential high-energy source by MunarAdrover et al. (2013), who using XMM-Newton observations, found that IRAS 16547-4247 is a hard X-ray source. They also found that may be difficult to explain the X-ray emission as non-thermal emission, but rather as thermal Bremsstrahlung plus photo-electric absorption in the cloud. At smaller scales close to the powering source, $0.85 \mathrm{~mm}$ continuum observations revealed that IRAS 16547-4247 is split into two compact sources, one of them spatially associated with IRAS 16547-4247, and the other one to the west (Zapata et al. 2015). In this way, Higuchi et al. (2015), using ${ }^{12} \mathrm{CO}(3-2)$ observations, detected two outflows, one of them aligned in the NW-SE direction, and the other one in the NE-SW direction, which suggest multiple driving sources powering outflows in the region.

In this paper we report GMRT observations of the jet associated with IRAS 165474247, aimed to explore for the first time the behavior of its associated shocks at very low frequencies, where effects such as synchrotron self-absorption and the low energy cut-off of the electron spectrum of the present electron population become important. Very low frequency studies (i.e. $<1 \mathrm{GHz}$ ) over proto-stellar jets are not frequent because, in addition to the low angular resolution (i.e. resulting in a poor astrometry) and terrestrial radio interference, contamination from the galactic background is notable. The observations and data reduction, including actions performed to overcome the mentioned difficulties, are described in Sect. 2 and in Sect. 3 we report the results. A discussion about our tentative findings is shown in Sect. 4. Finally, in Sect. 5 we summarize our work.

\section{Observations}

The GMRT observations were conducted in two observing blocks on 2017 Feb 21th and 28th. 3C286 was used as primary and bandpass calibrator, while J1714-252 was used for the gain calibration. The receivers were tuned at the $325 \mathrm{MHz}$ band and $610 \mathrm{MHz}$ band during the first and second day, respectively. The details of the observations are given in Table 1 .

The GMRT data were flagged, calibrated and imaged using the SPAM pipeline Intema et al. 2017) which includes several rounds of self-calibration and iterative flagging. Since the target is near the galactic plane, correction to the system temperature $T_{\text {sys }}$ arising due to excess background was needed. This correction was derived using the all-sky map at 408 $\mathrm{MHz}$ by Haslam et al. (1995) as part of SPAM pipeline. At $325 \mathrm{MHz}$, the Tsys correction factor applied was 6.10 and at $610 \mathrm{MHz}$, the $T_{\text {sys }}$ correction factor was 2.21.

At low frequencies, the ionosphere introduces some propagation delay differences be- 
tween the elements in the array. A method widely used to correct for these phase errors consists in performing self-calibration using several bright and compact sources in the initial image (Pearson \& Readhead 1984). An improved version of this scheme has been employed in the SPAM pipeline where the ionospheric corrections have been estimated for several directions. This scheme has been shown to produce significant improvement in image fidelity as compared to standard self-calibration (Intema et al. 2009). Though the self-calibration is well constrained, it could introduce small astrometric errors.

The astrometry of our data was checked using field sources from the GPSW catalog (White et al. 2005). From this catalogue, we employed sources 343.021+0.312, 343.144+0.032, 343.260-0.002 and 343.210-0.191 to obtain average offset corrections that were applied to our maps. In the source selection we intended to avoid multiplicity or extended emission. Our results are 0.4 and 3.7 arcsec to the west and north, respectively, for the $325 \mathrm{MHz}$ map, while for the 610 map the offsets are 1.2 and 5.1 arcsec to the east and north, respectively. The standard deviation of the corrections was $\sim 1$ arcsec. This uncertainty was added to the $10 \%$ of the beam size as the typical positional accuracy of GMRT. Furthermore, we checked these offsets by comparing our $610 \mathrm{MHz}$ map (before correction) with high frequency images at $4.8 \mathrm{GHz}$ (Rodríguez et al. 2005) convolved to our $610 \mathrm{MHz}$ beam and found consistent results. This can be assessed in Figure 1, where the knots at $610 \mathrm{MHz}$ appear displaced with respect to the $4.8 \mathrm{GHz}$ knots with an offset similar to that derived above. Because the emission peaks may slightly differ at low and high frequencies due to the contribution of different emission mechanisms, we adopted the offsets derived with point sources as the most accurate ones. After shifting the maps with the appropriate offsets, the sources belonging in the jet seen in our maps fall approximately in the jet axis derived in previous works.

\section{Results}

\subsection{Emission maps}

Fig. 2 shows the continuum emission of IRAS 16547-4247 at low radio frequencies. From these maps, the jet structure becomes evident as an elongated morphology with a size of $\sim 30^{\prime \prime} \times 20^{\prime \prime}$ in the SE-NW direction.

At $610 \mathrm{MHz}$, the jet shows three aligned continuum knots representing the triple radio source first detected by Garay et al. (2003). The northern knot is the brightest and the southern knot is the weakest and both sources are symmetrically separated $\sim 10^{\prime \prime}$ from the central source in opposite directions, in agreement with Garay et al. (2003). Two additional sources of $\sim 1$ mJy appear in the map about $15^{\prime \prime}$ East to the jet. While the southern source 
is located too far away to be considered related to the jet, the northern source could belong to the jet of we account for a wiggling motion. However, both sources are weak and were not reported before. Thus, they will not be further discussed in this work.

At $325 \mathrm{MHz}$, an elongated structure with two continuum knots separated $\sim 15^{\prime \prime}$ is revealed. While the location of the northern knot is nearly coincident with the position of the northern peak at $610 \mathrm{MHz}$, the southern knot can not be convincingly associated with any of the $610 \mathrm{MHz}$ counterparts. Similarly as for the $610 \mathrm{MHz}$ data, the northern knot seen at $325 \mathrm{MHz}$ is the brightest.

Figure 2 also shows the position of the peaks reported in Garay et al. (2003) using observations with similar angular resolution than our observations at $625 \mathrm{MHz}$ band. We measured the distance between the peak position of the northern and southern knots separately for the $325 \mathrm{MHz}$ and $610 \mathrm{MHz}$ bands. For a more exhaustive comparison, we performed the same measurement between the northern and southern peaks reported in Garay et al. (2003). We take the distance between the northern and southern peaks as representative of the jet size at each frequency. We obtained the following results: $20 \pm 0.1^{\prime \prime}$ (8.6 GHz, Garay et al. $2003), 19 \pm 1^{\prime \prime}(610 \mathrm{MHz})$ and $15 \pm 2^{\prime \prime}(325 \mathrm{MHz})$, whose uncertainties are estimated from the signal to noise ratio and beam size. Although the discrepancy in separation between knots at $8.6 \mathrm{GHz}$ and $610 \mathrm{MHz}$ is not significant, this separation estimated at $325 \mathrm{MHz}$ appears much shorter than in other bands. The derived sequence could suggest that the separation between the emission of the terminal shocks of the jet decreases at low frequencies.

The angular resolution of our $610 \mathrm{MHz}$ map is similar to that of the $1.4 \mathrm{GHz}$ map of Garay et al. (2003). However, the appearance of the latter map is different: the jet shows a northern peak with a tail extended to the south. The most plausible explanation on this relies on the position angle of the beam of the $610 \mathrm{MHz}$ map, that makes possible to resolve better the jet along its direction than the $1.4 \mathrm{GHz}$ map. To corroborate this, we degraded the angular resolution toward the direction of the jet in the $610 \mathrm{MHz}$ map and obtained the same jet appearance seen in the $1.4 \mathrm{GHz}$ map. On the other hand, in spite of the lower angular resolution of the $325 \mathrm{MHz}$ map with respect to the $610 \mathrm{MHz}$ map, the former shows a southern knot associated with the jet not seen at higher frequencies. According to the trend of the jet structure with frequency found above, this knot is not expected to be prominently detected if the emission in the jet behaves similarly at all frequencies. Thus, the $325 \mathrm{MHz}$ emission appears to arise from a different spot than the southern tip of the jet seen at higher frequencies. 


\subsection{Determination of spectral indices}

To extract fluxes from the maps we fitted to the jet emission three and two Gaussian models simultaneously for the $610 \mathrm{MHz}$ and $325 \mathrm{MHz}$ maps, respectively, with the CASA function fitcomponents from the CASA image toolkit. In order to prevent contamination of thermal emission from the central jet to the flux of the lobes at $325 \mathrm{MHz}$, a synthetic Gaussian model with the beam size at this frequency was subtracted to the corresponding position before performing the fits in the map. The thermal flux of the Gaussian model (1.3 mJy) was estimated from the fit of the central panel of Figure 3 that coincides with the $3-\sigma$ upper level for the $325 \mathrm{MHz}$ flux. The flux values obtained from the Gaussian fits are given in Table 2, together with the peak positions and the deconvolved source sizes. The central knot is unresolved indicating us that the very low frequency emission is not extended towards the central source, whereas the northern and southern knots have slightly extended sizes $\left(\sim 11^{\prime \prime} \times 4^{\prime \prime}\right)$. Nevertheless, these results are marginal and probably due to the fact that the lobes of the jet are composed of several smaller components as revealed by high angular resolution observations (Rodríguez et al. 2005, 2008).

Figure 3 shows the fit to the observed fluxes reported in the present work and in previous papers. In this fit we assumed that the emission have a power law dependence with frequency. From the literature, we selected data of Garay et al. (2003) as they employed observations with similar angular resolution than ours. The numerical results of these fits are reported in Table 3. As seen in the table, the total fit is obtained under a reasonably good correlation coefficient and our slope values do not differ significantly from those reported before (Garay et al. 2003; Rodríguez et al. 2005).

The dispersion seen in some points in the graphs is not crucial to corrupt our fit results and occurs often when comparing data with a wide time range. Flux density discrepancies between different observations can be attributed to differences in instrumental calibration or in $u v$-coverage. Otherwise, they can be due to flux variability inherent to thermal jets and HH knots, as they can show significant bright changes on timescales of few years (Rodríguez et al. 2000, 2001, Galván-Madrid et al. 2004). For instance, flux measurements for some of the knots of the HH $80 / 81 / 80 \mathrm{~N}$ jet have are discrepancies up to $50 \%$ between observations of different epochs (Martí et al. 1993; Masqué et al. 2012, 2015). In IRAS 16547-4247, some of the radio sources in the region show flux variations up to $40 \%$ (Rodríguez et al. 2008). For the particular case of the southern lobe, an increase with more than a factor of two in the $4.8 \mathrm{GHz}$ flux with respect to Garay et al. (2003) is observed, even though in this case we are comparing observations with different $u v$-coverage and, probably, the uncertainties are notable. 


\section{Discussion}

\subsection{Precession of the jet}

The angular separation of the northern and southern knots with the central source is similar $\left(\sim 10^{\prime \prime}\right)$, suggesting that the shocks that produce them can be associated to the same ejection event. However, as seen above, there are some differences between the northern and southern knots. First, the northern lobe is significantly brighter than the southern lobe. According to the inclination of the outflow axis from the line of sight of 84 degrees, with the red-shifted lobe pointing to the north, derived in Garay et al. (2007) the greater brightness at low radio frequencies observed for the corresponding knot is probably intrinsic. Second, as shown in Fig 2, our derived peak position at $325 \mathrm{MHz}$ for the southern knot is not fully coincident with the southern knot position seen at higher frequencies (e.g., Rodríguez et al. 2005).

It is interesting to note that the continuum peak position of the components of the jet are consistent between frequencies greater than $325 \mathrm{MHz}$ as observed by comparing between several works (Garay et al. 2003, Rodríguez et al. 2005, 2008) and our $610 \mathrm{MHz}$ map. This indicates us that their associated emission arises from the same shocks and that the knots lack for proper motions. On the other hand, the position of our knots at $325 \mathrm{MHz}$ shows evident discrepancies with these studies, suggesting that the very low frequency data make evident different characteristics in the shocks associated with the jet lobes. The peak position of the three knots detected at $610 \mathrm{MHz}$ (see Table 2) are aligned with a P.A. of $\sim 70^{\circ}$, similar to that of Rodríguez et al. (2005). On the other hand, at $325 \mathrm{MHz}$, the position of the northern knot has a position angle of $\sim 80^{\circ}$, still consistent with being part of the jet within uncertainties $\left(\sim 10^{\circ}\right)$, but the southern knot has a P.A. of $\sim 30^{\circ}$, which is similar to the uncertainty in P.A. for this knot. This rises the question whether this knot belongs to the jet or not.

Higuchi et al. (2015) found evidence for at least two intersecting outflows emanating from IRAS 16547-4247. Supported by the distribution of Class I methanol masers (Voronkov et al. 2006), they estimated the directions of the outflows as shown in their Figure 5. The location of the southern knot at $325 \mathrm{MHz}$ agrees with being part of the northeast-southwest outflow. However, the lack of additional radio counterparts for this outflow, added to the large uncertainty for our derived PA for the southern knot at $325 \mathrm{MHz}$, makes this possibility unlikely.

Alternatively, the detected knots at very low frequencies could represent other components of the jet reported in Rodríguez et al. (2005), which are not perfectly aligned due to the jet precession (Rodríguez et al. 2008). In Figure 4 , we show our maps with all the com- 
ponents of the jet marked with crosses and a precession model for the jet superimposed. The uncertainties in the alignment process added to the error in the position peak determination in the Gaussian fits are indicated in the figure. From this figure, we can see that the position of the knots reported in this paper falls approximately within the jet path and, hence, they are possibly tracing shocks as the precessing jet digs into the cloud. In this scenario, the 325 $\mathrm{MHz}$ emission would probe shocks with different properties along the jet. Furthermore, the somewhat curved morphology of the knots, suggests that even at very low frequencies, hints of precession can be inferred.

\subsection{Non-thermal emission from the lobes of the jet}

In Figure 3 we show the fits to the flux density of the knots of the jet reported in Table 2 assuming that they follow a power law dependence with frequency. We also considered that the emission of the southern $325 \mathrm{MHz}$ knot belongs to the southern lobe. Our results are in agreement with previous values obtained with a narrow bandwidth (Garay et al. 2003, Rodríguez et al. 2005) as indicated by the good correlation coefficient seen in Table 3 .

The radio emission of the jet lobes was proposed to arise from the interaction of the stellar jet with the surrounding medium. This interaction consists in shocks where a small fraction of the electrons are accelerated to relativistic velocities producing non-thermal emission if a magnetic field is present. In a similar study, Vig et al. (2018) found negative spectral indices for the knots of the $\mathrm{HH} 80 / 81 / 80 \mathrm{~N}$ jet steeper at the lowest frequencies observed over this jet $(325$ and $610 \mathrm{MHz}$ ) and was interpreted as thermal free-free contribution at higher frequencies. The lack of significant variations in our derived spectral indices along the 325 MHz- $8 \mathrm{GHz}$ range suggest that the contribution of thermal emission is not important at these frequencies. On the other hand, the spectral index found for the central source is positive, consistent with that derived in Garay et al. (2003) and Rodríguez et al. (2005), indicating that the emission is predominantly thermal.

As seen in Figure 4, the emission in the southern lobe at $325 \mathrm{MHz}$ appears to arise from a position different than the position traced by higher frequency data. We propose two possibilities: first, the southern $325 \mathrm{MHz}$ knot corresponds to a component aside from the jet. For instance, this knot could be associated with the time variable Source B (Rodríguez et al. 2005). Otherwise, the puzzling location of the southern knot at $325 \mathrm{MHz}$ could be representative of non-resolved substructure belonging to the southern lobe of the jet. Although the first possibility cannot be fully discarded, the good fit for the southern knot seen in Figure 3 favors the second possibility. Therefore, in the following discussion we will consider that the southern knot detected at $325 \mathrm{MHz}$ belongs to the jet. 
The high angular observations of Rodríguez et al. (2005) reveal that the lobes of the jet are composed by several components. The authors derived spectral indexes separately for these components and found variations between them, though most of the indices were negative. The angular resolution of our observations and those of Garay et al. (2003) implies that the fluxes of Fig. 3 include blended emission from different components and, hence, our spectral indices are representative of the average distribution of the relativistic electron population along the lobe. In this sense, the position of the southern emission peak at 325 $\mathrm{MHz}$ suggests that the electrons emitting mostly at these frequency are not concentrated on the southern tip of the shock, contrary to what occurs in the northern lobe.

Non-thermal emission is widely found in extragalactic objects such as quasars and radiogalaxies, that posses a central engine capable of launching relativistic jets. Differences in the emission of the expanding lobes depending on the observed frequency have been reported in extragalactic jets (e.g., Perley et al. 1984). Synchrotron decay due to electron cooling has been invoked to explain the differences observed between frequencies. A common assumption in the study of synchrotron emitting sources is the minimum energy condition that corresponds to equipartition of energy between relativistic particles and magnetic field. Following the prescription of Pacholczyk (1970), we can obtain an estimation of magnetic field in the lobes of the jet under this condition using the expression:

$$
\left[\frac{B_{\mathrm{eq}}}{\text { gauss }}\right]=5.69 \times 10^{-5}\left[\frac{1+\chi}{f(\sin \phi)^{3 / 2}}\left(\frac{\operatorname{arcsec}}{\theta_{x} \theta_{y}}\right)\left(\frac{\mathrm{kpc}}{s}\right) S_{\mathrm{rad}}\right]^{2 / 7}
$$

where $\chi$ is the energy ratio of the heavy particles to electrons for which we adopted 40 as measured in cosmic rays collected near the Earth (Simpson et al. 1983), $\phi$ is the angle between the magnetic field and the line of sight (we adopted $\sin \phi$ as 0.5 ), $s$ is the path length through the source in the line of sight that was estimated as $\sqrt{\theta_{x} \theta_{y}}$ and converted to linear size accounting for the distance to the source, $\alpha$ is the spectral index, whose values are reported in Table 3, $\theta_{x}$ and $\theta_{y}$ are the major and minor axis of the source size (we adopted $11^{\prime \prime} \times 4^{\prime \prime}$, see Table 2), $f$ is the filling factor of the source (assumed to be 0.5 ) and $S_{\text {rad }}$ is the total flux density integrated from 0.01 to $100 \mathrm{GHz}$ (e.g. Miley 1980) (typically between 0.04 and $0.05 \mathrm{Jy} \mathrm{beam}^{-1} \times \mathrm{GHz}$ ) that is obtained from the measured fluxes and spectral indices from Tables 2 and 3. From this expression, we obtain a rough estimate for the magnetic field of $\sim 0.5 \mathrm{mG}$. Despite obtaining a magnetic field value somewhat lower than the obtained by Rodríguez et al. (2008), both values are still consistent within the uncertainties inherent to our estimation and they fall within the range of values estimated in protostellar jets embedded in molecular clouds (0.1-1 mG: Carrasco-González et al. 2010, Ainsworth et al. 2014; Masqué et al. 2015; Vig et al. 2018). 
Following a formalism based on Longair (2011), the cooling time due to radiative processes for relativistic electrons, $t_{\mathrm{c}}$, is given by $t_{\mathrm{c}}(y r) \sim 2.3 \times 10^{4} \nu^{-1 / 2} B^{-3 / 2}$, where $\nu$ is our observing frequency in $\mathrm{Hz}$ and $B$ is the magnetic field given in Gauss. Using the $\mathrm{B}$ value estimated above, we get a $t_{\mathrm{c}}$ of $(8-10) \times 10^{4} \mathrm{yr}$ for our observed frequency values. This range encompasses the decay time value derived in Rodríguez et al. (2008) $\left(t_{\mathrm{d}}\right.$ of $\left.9 \times 10^{4} \mathrm{yr}\right)$. In fact, if we expand the range of decay time to all observable radio frequencies (up to $\sim 40$ $\mathrm{GHz}$ ) we get $\sim 10^{4-5} \mathrm{yr}$. According to the jet velocity (490 $\mathrm{km} \mathrm{s}^{-1}$, Rodríguez et al. 2008) we obtain a dynamical age of $280 \mathrm{yr}$. The much larger decay time for the synchrotron emission in the shocks makes unlikely the cooling mechanism for electrons as the shock evolves as responsible for the differences in the emission of the jet found between frequency bands.

Alternatively, different conditions of the associated shock/ambient properties of the southern lobe, with respect the northern lobe could be responsible for the observed discrepancies between frequencies. Contrary to extragalactic jets, protostellar jets dig into a denser inhomogeneous medium. Such density inhomogeneities are inherent to molecular clouds in a wide range of scales (e.g. Morata et al. 2003). In this scenario, the jet would impact on small unresolved clumps along the path with a variety of densities. Depending on the density of the ambient, the acceleration mechanism for electrons would be strength or inhibited. Then, the discrepancy of the appearance of $325 \mathrm{MHz}$ emission can be explained by collisions with the ambient gas at a given density, which could deplete the low energy electrons from the shock (Casillas-Pérez et al. 2016) from the tip of the southern lobe. This naturally explains the differences in brightness between the northern and southern lobes. In a similar recent work, although electron cooling was proposed as a possibility to explain flux variability observed in some knots in the HH 80/81/80N jet (Vig et al. 2018), the authors could not rule out associating the emission variability with the fact that the jet passes through an inhomogeneous medium. Indeed, Rodríguez et al. (2008) measured no significant proper motions for the knots and concluded that they are likely part of the working surfaces of the jet with the ambient medium. Therefore, the differences in emission between frequency bands and between the northern and southern lobes of the jet could be explained in therms of the interaction of the material of the jet with the medium through it advances, whose inhomogeneities affect the acceleration and depletion mechanisms for the electrons. Observations at mm wavelengths with higher angular resolution than those presented in this work could shed light on this possibility. 


\section{Summary}

The very low frequency radio emission $(325 \mathrm{MHz}$ and $610 \mathrm{MHz}$ ) of the jet associated with IRAS 16547-4247 have been explored. Despite detecting clearly the jet at these frequencies, important differences between frequency bands become evident. After accurately align the maps, we found that the knots seen at $325 \mathrm{MHz}$ are not totally coincident with the position of the knots associated with the jet seen at higher frequencies. We have calculated spectral indices by means of the integrated emission of the whole lobes and find consistent results with previous works showing that the non thermal emission is also present down to the observed frequencies. To explain the discrepant appearance of the jet between frequency bands, we discuss electron cooling in a magnetic field of $\sim 0.5 \mathrm{mG}$ as a possibility. However, the synchrotron decay time is much larger than the dynamical age of the jet. Alternatively,

we invoke interaction with an inhomogeneous medium as the most plausible scenario, though high resolution mm observations are required to assess this possibility.

The present work highlights the capability of very low frequency studies towards protostellar jets to explore some of its properties. At present, detailed simulations of the FokkerPlanck equation are being developed in order to extract conclusive results, which will be included in a forthcoming paper.

We thank Heinz Andernach for his crucial help with the astrometry of the maps. We are also grateful to Anabella Araudo for the fruitful comments on synchrotron losses.

\section{REFERENCES}

Ainsworth, R. E., Scaife, A. M. M., Ray, T. P., Taylor, A. M., Green, D. A., \& Buckle, J. V. 2014, ApJ, 792, L18

Anglada, G. 1996, in Astronomical Society of the Pacific Conference Series, Vol. 93, Radio Emission from the Stars and the Sun, ed. A. R. Taylor \& J. M. Paredes, 3-14

Anglada, G., Estalella, R., Mauersberger, R., Torrelles, J. M., Rodríguez, L. F., Canto, J., Ho, P. T. P., \& D'Alessio, P. 1995, ApJ, 443, 682

Anglada, G., López, R., Estalella, R., Masegosa, J., Riera, A., \& Raga, A. C. 2007, AJ, 133, 2799

Anglada, G., Rodríguez, L. F., \& Carrasco-González, C. 2018, A\&A Rev., 26, 3

Bally, J. 2016, ARA\&A, 54, 491 
Blandford, R. D., Begelman, M. C., \& Rees, M. J. 1982, Scientific American, 246, 124

Brooks, K. J., Garay, G., Mardones, D., \& Bronfman, L. 2003, ApJ, 594, L131

Carrasco-González, C., Rodríguez, L. F., Anglada, G., Martí, J., Torrelles, J. M., \& Osorio, M. 2010, Science, 330, 1209

Casillas-Pérez, G. A., Jeyakumar, S., Pérez-Enríquez, H. R., \& Trinidad, M. A. 2016, Advances in Space Research, 58, 1991

Castro, D. \& Slane, P. 2010, ApJ, 717, 372

Eislöffel, J., Smith, M. D., \& Davis, C. J. 2000, A\&A, 359, 1147

Fendt, C. 2006, ApJ, 651, 272

Galván-Madrid, R., Avila, R., \& Rodríguez, L. F. 2004, Rev. Mexicana Astron. Astrofis., 40, 31

Garay, G., Brooks, K. J., Mardones, D., \& Norris, R. P. 2003, ApJ, 587, 739

Garay, G., Mardones, D., Bronfman, L., Brooks, K. J., Rodríguez, L. F., Güsten, R., Nyman, L.-A., Franco-Hernández, R., \& Moran, J. M. 2007, A\&A, 463, 217

Girart, J. M., Curiel, S., Raymond, J., Rodrigues, L. F., \& Canto, J. 1996, in Astronomical Society of the Pacific Conference Series, Vol. 93, Radio Emission from the Stars and the Sun, ed. A. R. Taylor \& J. M. Paredes, 41

Girart, J. M., Curiel, S., Rodríguez, L. F., \& Cantó, J. 2002, Rev. Mexicana Astron. Astrofis., 38, 169

Girart, J. M., Fernández-López, M., Li, Z.-Y., Yang, H., Estalella, R., Anglada, G., Áñez-López, N., Busquet, G., Carrasco-González, C., Curiel, S., Galvan-Madrid, R., Gómez, J. F., de Gregorio-Monsalvo, I., Jiménez-Serra, I., Krasnopolsky, R., Martí, J., Osorio, M., Padovani, M., Rao, R., Rodríguez, L. F., \& Torrelles, J. M. 2018, ApJ, 856, L27

Haslam, C. G. T., Salter, C. J., Stoffel, H., \& Wilson, W. E. 1995, Astronomy Data Image Library

Higuchi, A. E., Saigo, K., Chibueze, J. O., Sanhueza, P., Takakuwa, S., \& Garay, G. 2015, ApJ, 798, L33

Intema, H. T., Jagannathan, P., Mooley, K. P., \& Frail, D. A. 2017, A\&A, 598, A78 
Intema, H. T., van der Tol, S., Cotton, W. D., Cohen, A. S., van Bemmel, I. M., \& Röttgering, H. J. A. 2009, A\&A, 501, 1185

Longair, M. S. 2011, High Energy Astrophysics

López, R., Estalella, R., Gómez, G., Riera, A., \& Carrasco-González, C. 2009, A\&A, 498, 761

López-Santiago, J., Peri, C. S., Bonito, R., Miceli, M., Albacete-Colombo, J. F., Benaglia, P., \& de Castro, E. 2013, ApJ, 776, L22

Martí, J., Rodríguez, L. F., \& Reipurth, B. 1993, ApJ, 416, 208

-. 1995, ApJ, 449, 184

-. 1998, ApJ, 502, 337

Masqué, J. M., Girart, J. M., Estalella, R., Rodríguez, L. F., \& Beltrán, M. T. 2012, ApJ, 758, L10

Masqué, J. M., Rodríguez, L. F., Araudo, A., Estalella, R., Carrasco-González, C., Anglada, G., Girart, J. M., \& Osorio, M. 2015, ApJ, 814, 44

Miley, G. 1980, ARA\&A, 18, 165

Morata, O., Girart, J. M., \& Estalella, R. 2003, A\&A, 397, 181

Munar-Adrover, P., Bosch-Ramon, V., Paredes, J. M., \& Iwasawa, K. 2013, A\&A, 559, A13

Ouyed, R. \& Pudritz, R. E. 1997, ApJ, 482, 712

Pacholczyk, A. G. 1970, Radio astrophysics. Nonthermal processes in galactic and extragalactic sources

Pearson, T. J. \& Readhead, A. C. S. 1984, ARA\&A, 22, 97

Perley, R. A., Dreher, J. W., \& Cowan, J. J. 1984, ApJ, 285, L35

Pudritz, R. E., Ouyed, R., Fendt, C., \& Brandenburg, A. 2007, Protostars and Planets V, 277

Raga, A. \& Cabrit, S. 1993, A\&A, 278, 267 
Raga, A. C., Velázquez, P. F., de Gouveia dal Pino, E. M., Noriega-Crespo, A., \& Mininni, P. 2003, in Revista Mexicana de Astronomia y Astrofisica, vol. 27, Vol. 15, Revista Mexicana de Astronomia y Astrofisica Conference Series, ed. J. Arthur \& W. J. Henney, $115-119$

Reipurth, B. \& Bally, J. 2001, ARA\&A, 39, 403

Reynolds, S. P. 1986, ApJ, 304, 713

Rodríguez, L. F., Delgado-Arellano, V. G., Gómez, Y., Reipurth, B., Torrelles, J. M., Noriega-Crespo, A., Raga, A. C., \& Cantó, J. 2000, AJ, 119, 882

Rodríguez, L. F., Garay, G., Brooks, K. J., \& Mardones, D. 2005, ApJ, 626, 953

Rodríguez, L. F., Ho, P. T. P., Torrelles, J. M., Curiel, S., \& Canto, J. 1990, ApJ, 352, 645

Rodríguez, L. F., Moran, J. M., Franco-Hernández, R., Garay, G., Brooks, K. J., \& Mardones, D. 2008, AJ, 135, 2370

Rodríguez, L. F. \& Reipurth, B. 1989, Rev. Mexicana Astron. Astrofis., 17, 59

Rodríguez, L. F., Torrelles, J. M., Anglada, G., \& Martí, J. 2001, Rev. Mexicana Astron. Astrofis., 37, 95

Shu, F. H., Adams, F. C., \& Lizano, S. 1987, ARA\&A, 25, 23

Simpson, J. A., Wefel, J. P., \& Zamow, R. 1983, International Cosmic Ray Conference, 10, 322

Vig, S., Veena, V. S., Mandal, S., Tej, A., \& Ghosh, S. K. 2018, MNRAS, 474, 3808

Voronkov, M. A., Brooks, K. J., Sobolev, A. M., Ellingsen, S. P., Ostrovskii, A. B., \& Caswell, J. L. 2006, MNRAS, 373, 411

Walmsley, M. 1995, in Revista Mexicana de Astronomia y Astrofisica, vol. 27, Vol. 1, Revista Mexicana de Astronomia y Astrofisica Conference Series, ed. S. Lizano \& J. M. Torrelles, 137

White, R. L., Becker, R. H., \& Helfand, D. J. 2005, AJ, 130, 586

Wilner, D. J., Reid, M. J., \& Menten, K. M. 1999, ApJ, 513, 775

Zanni, C., Ferrari, A., Rosner, R., Bodo, G., \& Massaglia, S. 2007, A\&A, 469, 811 
Zapata, L. A., Palau, A., Galván-Madrid, R., Rodríguez, L. F., Garay, G., Moran, J. M., \& Franco-Hernández, R. 2015, MNRAS, 447, 1826

This preprint was prepared with the AAS LATEX macros v5.2. 
Table 1: Details of the GMRT observations

\begin{tabular}{cccccc}
\hline \hline $\begin{array}{c}\text { Frequency } \\
(\mathrm{MHz})\end{array}$ & $\begin{array}{c}\text { Observation } \\
\text { date }\end{array}$ & $\begin{array}{c}\text { Bandwidth } \\
(\mathrm{MHz})\end{array}$ & $\begin{array}{c}\text { synthesized beam } \\
\left({ }^{\prime \prime} \times \times^{\prime \prime}\right)\end{array}$ & $\begin{array}{c}\sigma_{\text {rms }}^{a} \\
\text { mJy beam }^{-1}\end{array}$ \\
\hline 325 & 2017 Feb 21 & 32 & $24.2 \times 5.8$ & 12.2 & 0.6 \\
610 & 2017 Feb 28 & 32 & $12.4 \times 3.3$ & 15.6 & 0.3 \\
\hline
\end{tabular}

${ }^{a}$ Measured around the phase reference center, where the jet is found 


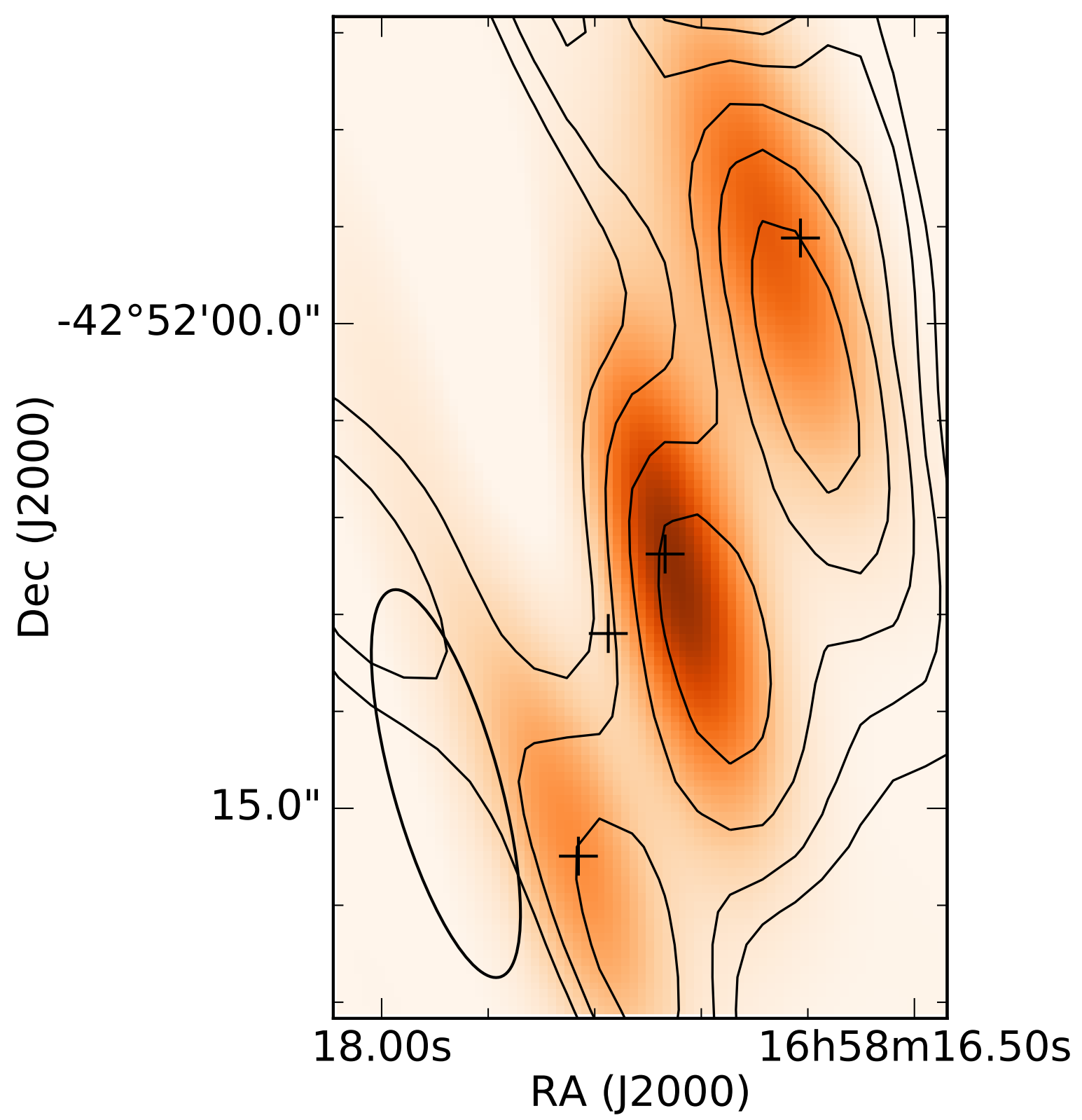

Fig. 1.- Comparison of the map obtained at $610 \mathrm{MHz}$ (contours) before performing the astrometric correction (see text) with the $4.8 \mathrm{GHz}$ data of Rodríguez et al. (2005) convolved to the GMRT $610 \mathrm{MHz}$ beam size (color scale). Contours are $C$ times $-2^{0}, 2^{0}, 2^{1 / 2}, 2^{1}, 2^{3 / 2}$ and $2^{2}$, where $C$ is given by 2.5 times the $r m s$ noise of the map $\left(0.3 \mathrm{mJy}_{\text {beam }}{ }^{-1}\right)$. The beam is shown in the bottom left corner. Although we corrected the astrometry of our data using field sources, this figure is shown to illustrate the displacement of our maps with respect other observations and how our offset corrections are adequate. 


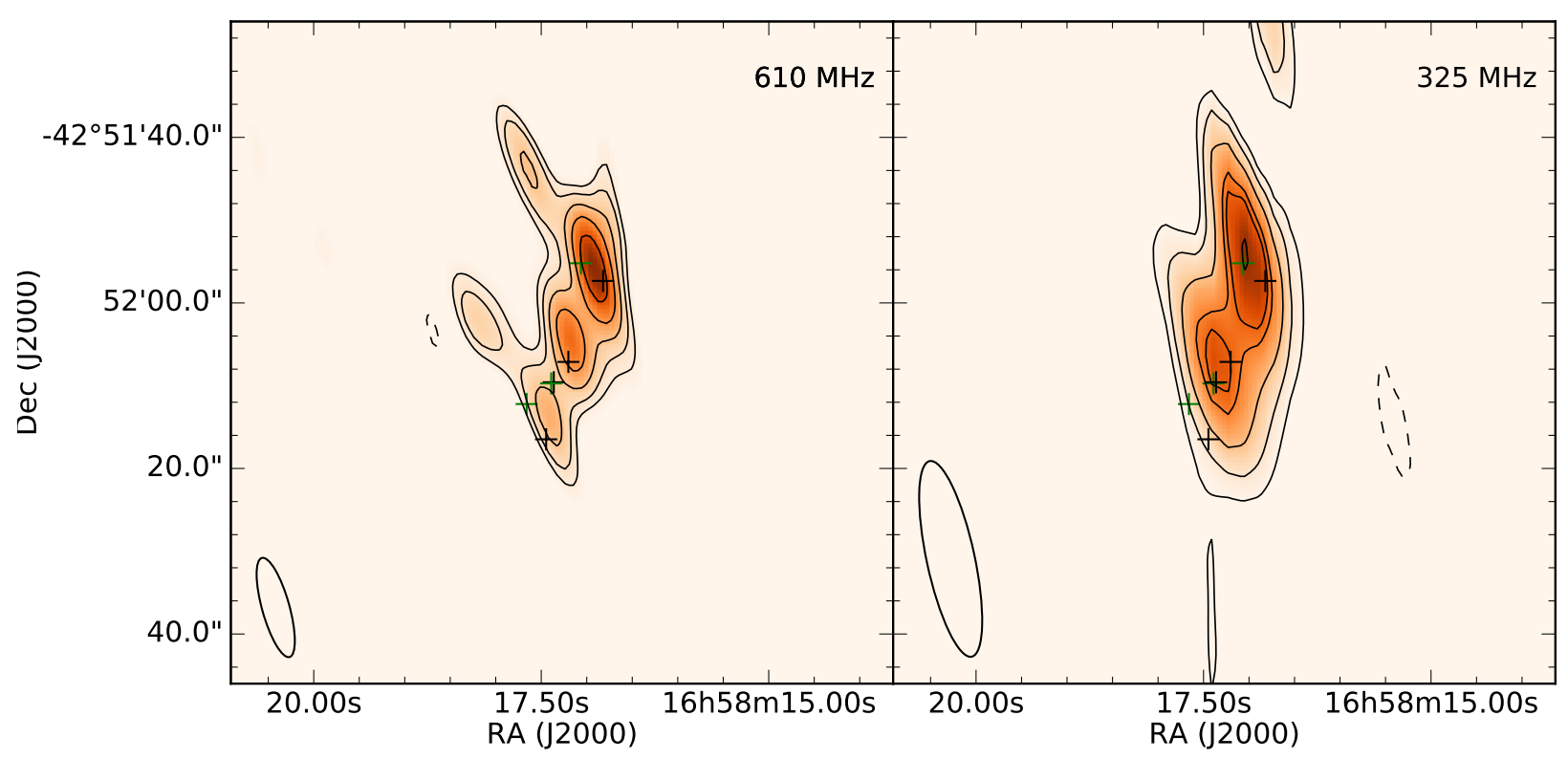

Fig. 2.- GMRT continuum emission maps at $325 \mathrm{MHz}$ (right panel) and $610 \mathrm{MHz}$ (left panel) of IRAS 16547-4247 (color and contours) after the astrometric correction. Contours are $C$ times $-2^{0}, 2^{0}, 2^{1 / 2}, 2^{1}, 2^{3 / 2}, 2^{2}$ and $2^{5 / 2}$, where $C$ is given by 2.5 times the $r m s$ noise of each map (0.6 mJy beam ${ }^{-1}$ for $325 \mathrm{MHz}$ and $0.3 \mathrm{mJy}_{\text {beam }}{ }^{-1}$ for $610 \mathrm{MHz}$ ). Black crosses represent the peak position of the knots derived in Garay et al. (2003), while cyan crosses represent the sources sources that are outside of the jet path derived in Rodríguez et al. (2005). The beam is shown in the bottom left corner. 


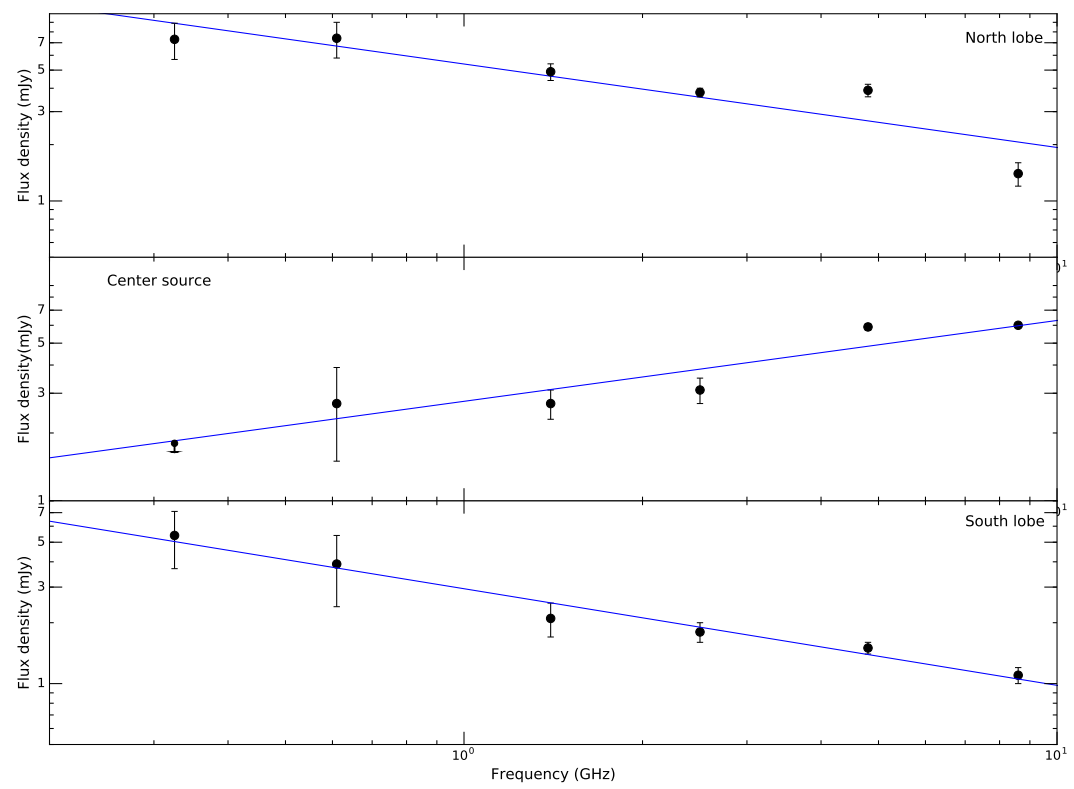

Fig. 3.- Plots of the flux density vs frequency of the northern lobe (top), central source (middle) and southern lobe (bottom) using the observed data Garay et al. (2003) and ours (black dots and error bars). The blue solid line shows the best fit to the observed fluxes assuming a power law dependence with the frequency. The arrow in the middle panel at 325 $\mathrm{MHz}$ represents 3 times the rms noise level. 


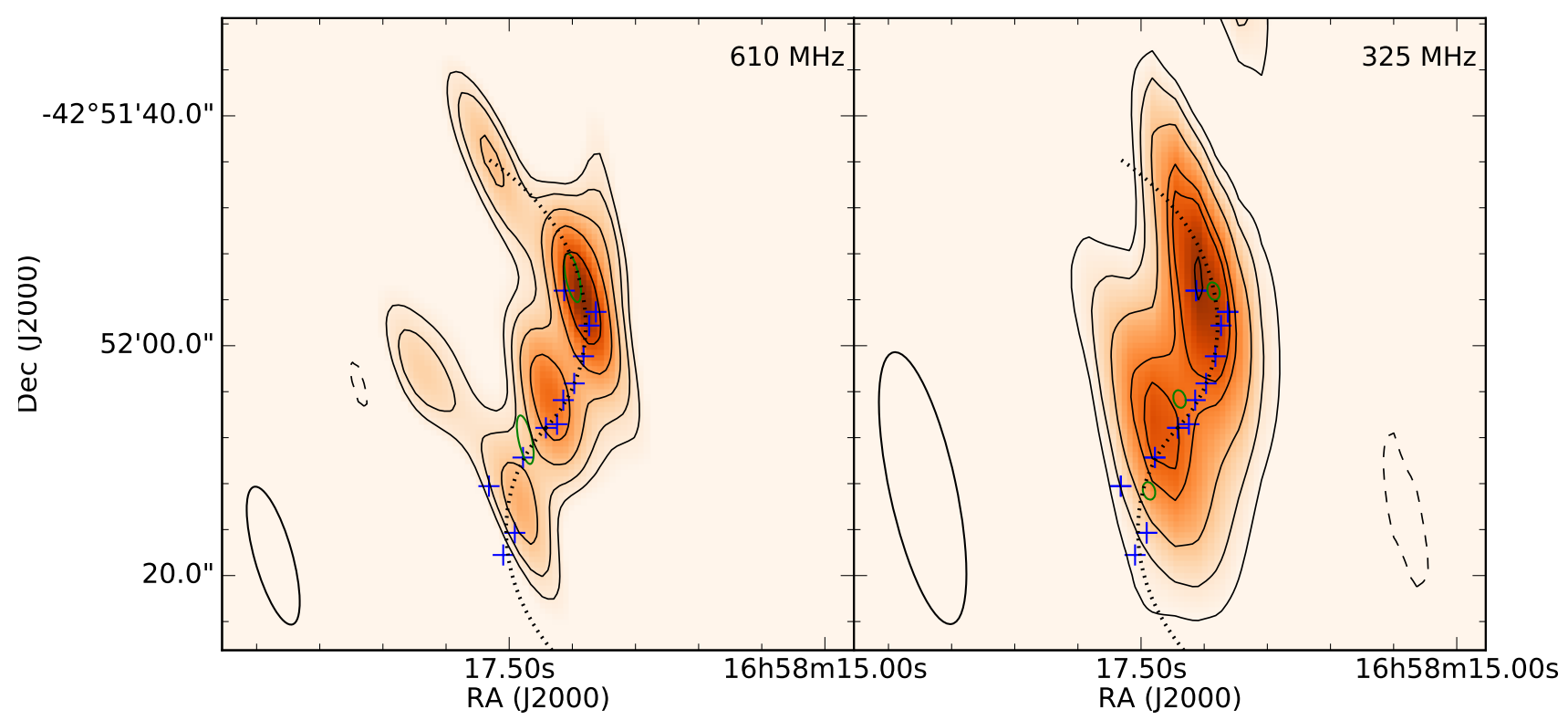

Fig. 4.- GMRT continuum emission maps at $325 \mathrm{MHz}$ (right panel) and $610 \mathrm{MHz}$ (left panel) of IRAS 16547-4247 (color and contours). Contours are the same as in Fig. 2 . The blue crosses represent the peak positions of all the outflow sources observed in 2006 (Rodríguez et al. 2008). The green circles represent the peaks resulting from Gaussian fits to the data and show the uncertainties in the alignment process (see text). In order to compare the position of the knots at both frequencies, we show the ellipses corresponding to the 325 $\mathrm{MHz}$ peaks in the $610 \mathrm{MHz}$ map and the other way around for the $325 \mathrm{MHz}$ map. The dashed line represents the spiral model employed in Rodríguez et al. (2008). 


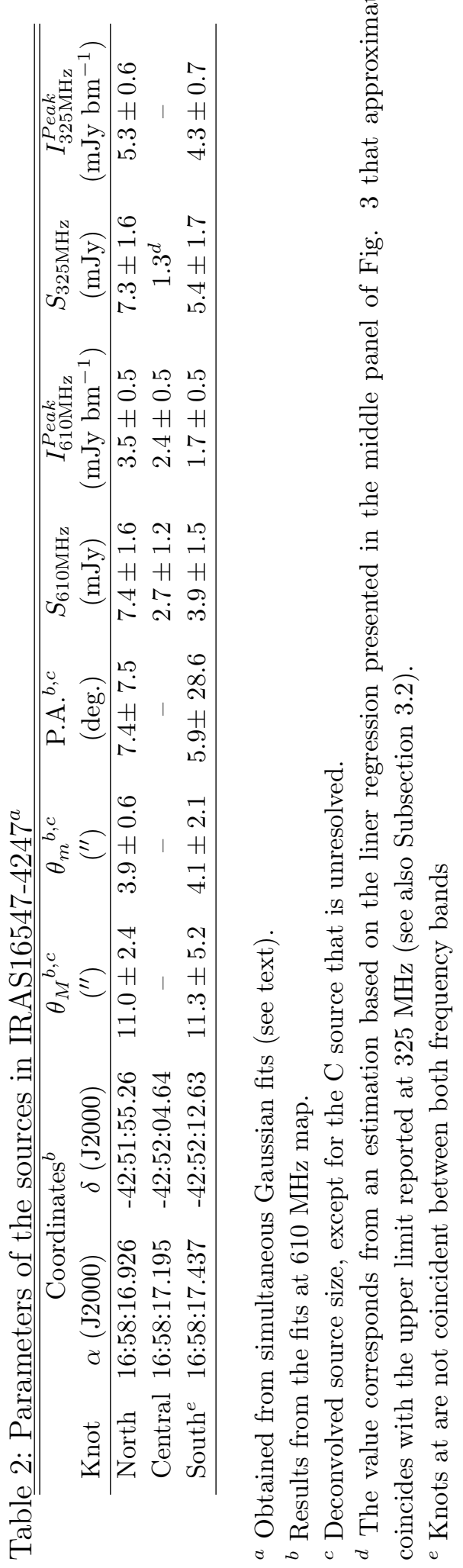




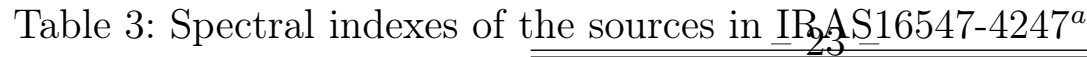

\begin{tabular}{ccc}
\hline \hline Source & $\alpha^{a}$ & $r^{2}{ }^{b}$ \\
\hline Northern lobe & $-0.45 \pm 0.10$ & 0.81 \\
Center & $0.44 \pm 0.08$ & 0.87 \\
Southern lobe & $-0.48 \pm 0.04$ & 0.97 \\
\hline
\end{tabular}

${ }^{a}$ Spectral index obtained including data points from Garay et al. (2003) and the present work. We excluded some data points (see text).

${ }^{b}$ Square of correlation coefficient. 